ARTICLE

\title{
Ultra-sensitive nanometric flat laser prints for binocular stereoscopic image
}

Dejiao Hu ${ }^{1,4}$, Hao Li ${ }^{1,4}$, Yupeng Zhu², Yuqiu Lei ${ }^{1}$, Jing Han ${ }^{1}$, Shilin Xian², Jiajin Zheng ${ }^{3}$, Bai-Ou Guan', Yaoyu Cao ${ }^{1 凶}$, Lei Bi ${ }^{2 \otimes} \&$ Xiangping Li (iD ${ }^{1 凶}$

Two-dimensional (2D) transition metal dichalcogenides (TMDs) with tantalizing layerdependent electronic and optical properties have emerged as a paradigm for integrated flat opto-electronic devices, but their widespread applications are hampered by challenges in deterministic fabrication with demanded shapes and thicknesses, as well as light field manipulation in such atomic-thick layers with negligible thicknesses compared to the wavelength. Here we demonstrate ultra-sensitive light field manipulation in full visible ranges based on $\mathrm{MoS}_{2}$ laser prints exfoliated with nanometric precisions. The nontrivial interfacial phase shifts stemming from the unique dispersion of $\mathrm{MoS}_{2}$ layers integrated on the metallic substrate empower an ultra-sensitive resonance manipulation up to $13.95 \mathrm{~nm}$ per $\mathrm{MoS}_{2}$ layer across the entire visible bands, which is up to one-order-of-magnitude larger than their counterparts. The interlayer van der Waals interactions and the anisotropic thermal conductivity of layered $\mathrm{MoS}_{2}$ films endow a laser exfoliation method for on-demand patterning $\mathrm{MoS}_{2}$ with atomic thickness precision and subwavelength feature sizes. With this, nanometric flat color prints and further amplitude-modulated diffractive components for binocular stereoscopic images can be realized in a facile and lithography-free fashion. Our results with demonstrated practicality unlock the potentials of, and pave the way for, widespread applications of emerging 2D flat optics.

\footnotetext{
${ }^{1}$ Guangdong Provincial Key Laboratory of Optical Fiber Sensing and Communications, Institute of Photonics Technology, Jinan University, Guangzhou, China.

${ }^{2}$ National Engineering Research Center of Electromagnetic Radiation Control Materials, University of Electronic Science and Technology of China, Chengdu, China. ${ }^{3}$ College of Electronic and Optical Engineering, Nanjing University of Posts \& Telecommunications, Nanjing, China. ${ }^{4}$ These authors

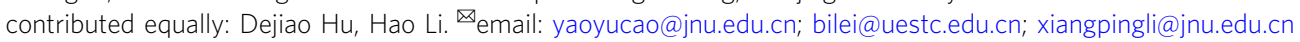


S ince the first discovery ${ }^{1,2}$, two-dimensional (2D) materials with exceptional optical and electronic properties have offered an unprecedented platform for developing ultracompact flat opto-electronic devices with a considerable degree of miniaturization. Transition metal dichalcogenides (TMDs) ${ }^{3,4}$ exhibiting sensitive layer-dependent properties, including indirect-to-direct bandgap transition from bulk states to monolayers, emerge as a peculiar complementary to graphene for investigating excitonic light-matter interactions. As such, onrushing developments of extensive applications have been witnessed in photodetectors ${ }^{5,6}$, valley emissions ${ }^{7}$, transistors ${ }^{8,9}$, and memories ${ }^{10,11}$.

In spite of manifesting enticing high refractive indices ${ }^{12,13}$, the light field manipulation in nanometric TMD layers remains dull sensitivity to their vanishing small thickness compared to the wavelength of operation. Until very recently, nanostructured TMD layers at elevated thicknesses to hundreds of nanometres are starting to be appreciated as high-index dielectric resonators supporting distinct geometry-dependent Mie resonances, which starts a research to shaping light's wavefronts by $2 \mathrm{D}$ materials based optical elements ${ }^{14,15}$. However, these demonstrations are achieved at the cost of degraded device compactness and integration. Moreover, the fabrication of nanostructured TMD layers mainly relies on mechanical or chemical exfoliation from bulk materials ${ }^{16,17}$ and subsequently follows complex lithography procedures to produce demanded shapes and thicknesses ${ }^{14,15}$. Even though these approaches are demonstrated effective for fundamental researches, aforementioned challenges remain the major hurdle for the pragmatic and widespread applications of the emerging $2 \mathrm{D}$ flat optics.

Here, we demonstrate ultra-sensitive light field manipulation in resonance spectra by nanometrically maneuvering thickness of $\mathrm{MoS}_{2}$ layers through a laser exfoliation technique. The anisotropic thermal conductivity of layered $\mathrm{MoS}_{2}$ films integrated on a metallic substrate with two orders of magnitude difference in inplane and out-of-plane directions leads to a giant temperature gradient along stacking layers. Combing the interlayer van der Waals interactions, it enables layer-by-layer laser exfoliation effects $^{18,19}$ which provide a pragmatic and lithography-free means to on-demand structure $\mathrm{MoS}_{2}$ flakes at atomic layer precisions and subwavelength feature sizes. By introducing the giant interfacial phase shifts associated with imaginary parts of their refractive indices, the resonance exhibiting ultra-sensitivity to the nanometric thickness can lead to not only vivid color appearances in full visible ranges but also large reflectance amplitude modulations. The proof-of-principle demonstration of flat color prints and further amplitude-modulated diffractive components for multi-perspective stereoscopic images unfolds the potential of $2 \mathrm{D}$ flat optics with practicality and up-scalability.

\section{Results}

Ultra-sensitive resonance manipulation through laser exfoliating nanometric $\mathbf{M o S}_{\mathbf{2}}$ layers. The typical configuration of nanometric $\mathrm{MoS}_{2}$ multilayers integrated on a gold substrate for ultra-sensitive resonance manipulation by direct laser writing technique 20,21 is schematically illustrated in Fig. 1. The $\mathrm{MoS}_{2}$ thin films prepared through pulsed laser deposition with an initial thickness of $20 \mathrm{~nm}$ were deposited on a gold substrate (see "Methods"). The layered crystal structure of as-grown $\mathrm{MoS}_{2}$ thin films was verified by both X-ray diffraction (Supplementary Fig. 1) and high-resolution transmission electron microscopy (Supplementary Fig. 2) experiments. The optical constants from the ultraviolet to near-infrared wavelength range were obtained using spectroscopic ellipsometry (Supplementary Fig. 3), which are used for calculations throughout the paper. A continuous wave $(\mathrm{CW})$ laser beam at the wavelength of $532 \mathrm{~nm}$ was focused by an objective lens $(\times 50, \mathrm{NA}=0.75)$ to pattern $\mathrm{MoS}_{2}$ layers. Light absorption in the upper layers can produce a local temperature rise that burns out atoms in the vicinity of the focal region, which can be dexterously controlled by the laser recipe. Figure $1 \mathrm{~b}$ shows the reflection optical image of a tangram pattern fabricated through the laser writing method. The zones with distinct color appearances represent $\mathrm{MoS}_{2}$ thin films with different nanometric thicknesses, verified by the topographic atomic force microscopy (AFM) image. Indeed, the structure exhibits extreme sensitivity in resonance tunability across in the full visible range through subtle variations of the thickness of $\mathrm{MoS}_{2}$ thin films by merely tens of atomic layers.

To gain insights into the ultra-sensitive resonance manipulation, we first review the Fabry-Perot (FP) resonance support by a thin film. FP resonance modes are standing waves formed by light wave propagating back and forth between two reflective interfaces, where constructive interference occurs after the light wave travels a round-trip. The resonance condition can be matched once the total phase accumulation, including both propagation phase shifts and interfacial phase retardations acquired at interfaces to be an integer modulus of $2 \pi$. Thus, the resonance wavelength can be simply derived as

$$
\lambda=\frac{2 n}{m-\left(\frac{\varphi_{1}+\varphi_{2}}{2 \pi}\right)} h
$$

where $n$ is the real part of the refractive index of the FP layer, $h$ is its thickness, $m$ is the order of resonance, and $\varphi_{1}$ and $\varphi_{2}$ are interfacial phase shifts defined to lie in the interval $[0,2 \pi)$ at the two interfaces, respectively. It can be seen that the sensitivity of the resonance wavelength to the FP thickness is mainly governed by the refractive index of the material and total interfacial phase shifts at the two interfaces. Especially, when the total interfacial phase shifts are reaching $2 \pi$, the denominator will approach zero for the first order resonance $(m=1)$. In this case, the resonance wavelength will be ultra-sensitive to even nanometric thicknesses of the FP medium, which leads to performances superior to conventional optical coatings relying on the propagation phase accumulation in a quarter-wave-thick film ${ }^{22}$ (Supplementary Note and Supplementary Fig. 4).

To realize such ultra-sensitive resonance manipulation, dielectric thin films with large complex indices as well as proper substrate designs are of vital importance. The unique dispersion of $\mathrm{MoS}_{2}$ thin films integrated on the $\mathrm{Au}$ substrate empowers broadband interfacial phase shifts reaching $2 \pi$ in the visible regime (Fig. 1c). Consequently, the largest tunability in reflectance spectra corresponding to a wide color palette by varying nanometric thickness of $\mathrm{MoS}_{2}$ layers can be realized, which exceedingly outperforms the other substrates such as Ag and Silicon (Supplementary Fig. 5). Figure 1d depicts the theoretical calculation results of the reflectivity spectra of the $\mathrm{MoS}_{2}-\mathrm{Au}$ structure with different numbers of layers (see "Methods"). As can already be inferred from the white dotted line marking out the evolution of resonance wavelengths, nearly $400 \mathrm{~nm}$ shift from $800 \mathrm{~nm}$ to $430 \mathrm{~nm}$ covering the entire visible light can be obtained by a thickness variation from 30 layers to a monolayer. It is worth noting that even the extinction-associated interfacial phase shifts ${ }^{23}$ are ubiquitous among dissipative materials with a large imaginary part of refractive index, the enticing dispersion properties of $\mathrm{MoS}_{2}$ thin films covering the outermost range in the complex index diagram (Fig. 1c) manifest a far superior sensitivity (defined as resonance wavelength shifts divided by the number of layers) of $13.95 \mathrm{~nm}$ per $\mathrm{MoS}_{2}$ layer, which shows up to 6 times and 2 times greater than that of $2.3 \mathrm{~nm}$ 


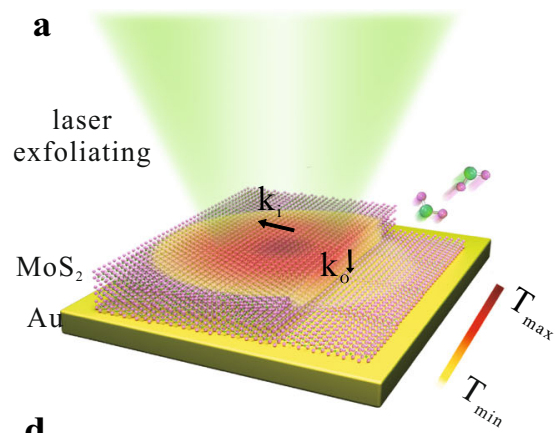

d

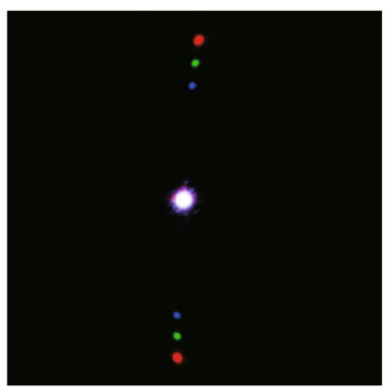

b

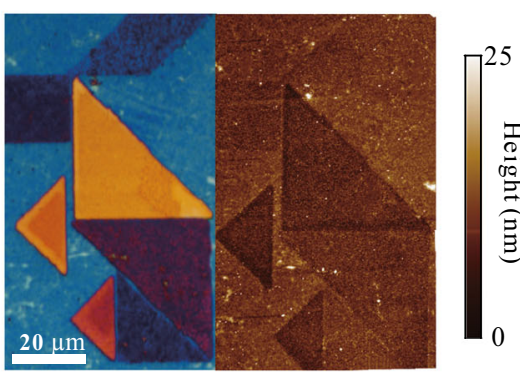

f

e

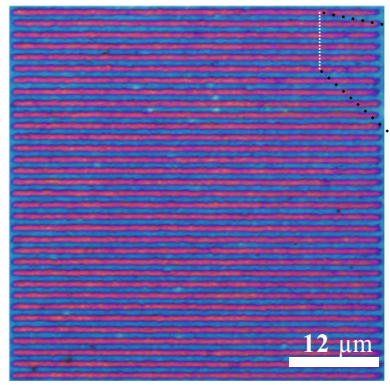

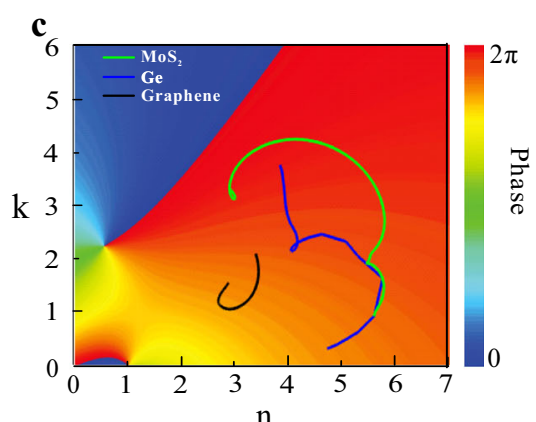

$\mathbf{h}$

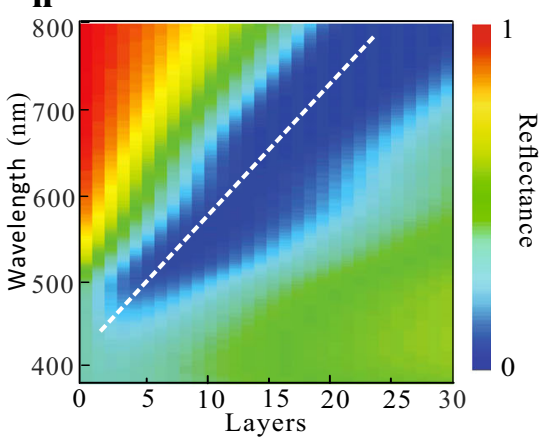

Fig. 1 Ultra-sensitive resonance manipulation by laser exfoliating $\mathbf{M o S}_{\mathbf{2}}$ thin films. a Schematic diagram of tightly focused laser beams for exfoliating

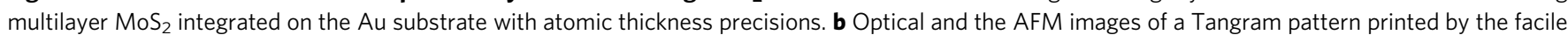
laser writing method. c The calculated total interfacial phase shifts for a dielectric layer-Au configuration with variant complex refractive index overlaid with the complex refractive index diagram of $\mathrm{MoS}_{2}$ (green), graphene (black), and Ge (blue). $\mathbf{d}$ The calculated reflectance spectra of the MoS 2 thin films on the Au substrate with different numbers of layers. e Microscopic image of the grating structure fabricated with a period of 1800 nm. $\mathbf{f}$ Surface corrugation curve of patterned nanometric gratings corresponding to the dotted line. $\mathbf{g}$ Reflectance amplitude modulations by such grating structures. $\mathbf{h}$ Diffraction image retrieved by using three beams at wavelengths of 450,550, and $650 \mathrm{~nm}$ simultaneously.

per graphene layer and $5.9 \mathrm{~nm}$ per Ge layer, respectively (Supplementary Fig. 6).

The ultra-sensitivity of resonances to the nanometric thickness can lead to not only full visible spectral tuning but also large reflectance amplitude modulations. Close to the resonance wavelength of $532 \mathrm{~nm}$, a $5 \mathrm{~nm}$-thick $\mathrm{MoS}_{2}$ film corresponding to 7 atomic layers can lead to nearly vanishing reflectance, while a $20 \mathrm{~nm}$-thick film can shift the resonance away, leading to an enhanced reflectance approaching 0.5 . As such, laser patterned nanometric flat grating structures (Fig. 1e) with a surface corrugation of $15 \mathrm{~nm}$ (Fig. 1f) are capable of light field manipulation based on strong amplitude modulation strength up to 0.44 (Fig. 1g). As an illustration, the diffraction patterns under 450, 550, and $650 \mathrm{~nm}$ lasers simultaneous illumination unambiguously verify the light field manipulation by such nanometric flat gratings (Fig. $1 \mathrm{~h}$ ).

Apparently, the interlayer interaction by van der Waals forces results in the dominant heat dissipation along the in-plane direction rather than the out-of-plane direction ${ }^{18,24}$. The layered $\mathrm{MoS}_{2}$ films on the Au substrate exhibit two orders of magnitude difference in thermal conductivity along the in-plane and out-ofplane directions, which can result in a giant temperature gradient along stacking layers (Supplementary Fig. 7). The local temperature in the upper layers is quickly built up to exceed the burning temperature and the beneath layers are less susceptible unless irradiation at high powers. This provides the basis for reliable thickness control at atomic layer precisions by establishing a standardized laser exfoliating recipe. Figure $2 \mathrm{a}-\mathrm{d}$ illustrate the systematic investigation of nanometric thickness control at variant laser doses, and characterization obtained through AFM and Raman spectroscopy (see "Methods"). The laser scanning speed is optimized and fixed at $0.1 \mathrm{~mm} / \mathrm{s}$ in the whole experiment (see Methods). By varying the laser irradiance powers with an increment of $8 \mathrm{~mW}$, the thickness of $\mathrm{MoS}_{2}$ thin films can be thinned in a staircase behavior with a step height of approximately $3 \mathrm{~nm}$ corresponding to 4 layers (given the monolayer thickness about $0.67 \mathrm{~nm}^{25}$ ). The surface roughness is on the order of $7.491 \mathrm{~nm}$ due to the presence of splashed or unremoved residuals, albeit no significant influence on their light field manipulation capabilities.

Since the frequency interval between $E_{2 g}$ and $A_{1 g}$ Raman modes depends monotonically on the number of $\mathrm{MoS}_{2}$ layers ${ }^{26,27}$, Raman spectroscopy is utilized to characterize the laser exfoliation process. Figure $2 \mathrm{c}$ depicts the evolution of Raman spectroscopy during the laser exfoliation. Initially, the frequency interval is $26 \mathrm{~cm}^{-1}$ for the $20 \mathrm{~nm}$ thick flakes indicating a multilayer state. As the laser dose increases, the frequency interval monotonically decreases close to $21 \mathrm{~cm}^{-1}$ implying a monolayer to bilayer state. The above characterization consolidates the effectiveness of the laser writing technique for thickness control at atomic precisions as well as up-scalability for on-demand fabricating layered $\mathrm{MoS}_{2}$ based optical elements.

From Eq. (1), it clearly reveals that the nanometric thickness variation of $\mathrm{MoS}_{2}$ thin films integrated on the Au substrate leads to an ultra-sensitive light field management in resonance wavelengths. Figure $2 \mathrm{e}$ and $\mathrm{f}$ show the theoretical calculation results of reflection spectra from that structure at variant nanometric thicknesses of $\mathrm{MoS}_{2}$ thin films as well as corresponding experimental results. The experiments are in good congruence with the theoretical predictions. It can be seen that the reflection valley caused by the resonant absorption can be continuously tuned in the whole range of visible light from the wavelength of more than $700 \mathrm{~nm}$ to $500 \mathrm{~nm}$, by merely a thickness decrease from $\sim 20 \mathrm{~nm}$ to $5 \mathrm{~nm}$. Optical micrographs of the colors generated from the laser thinned zones and corresponding color coordinate map are shown in Fig. $2 \mathrm{~g}$, h, respectively. 
a

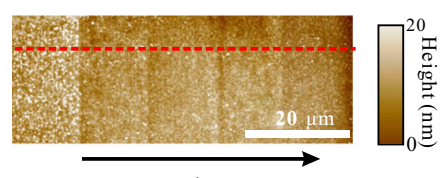

Increasing Power
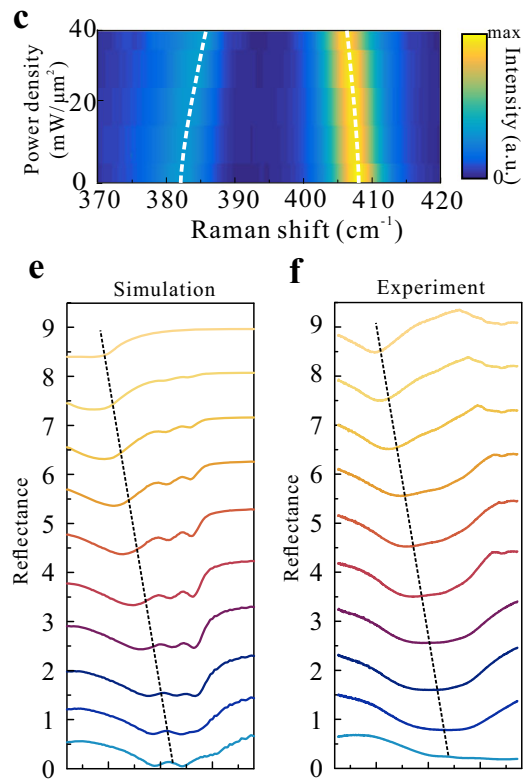

500600700 Wavelength $(\mathrm{nm})$ f
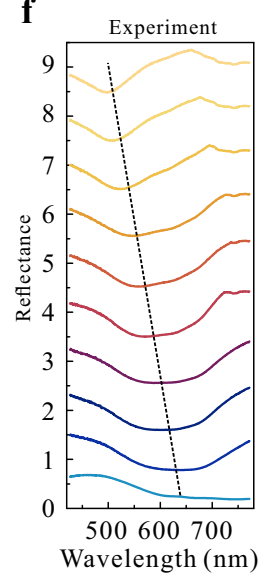

b

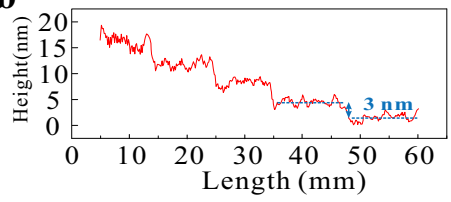

d

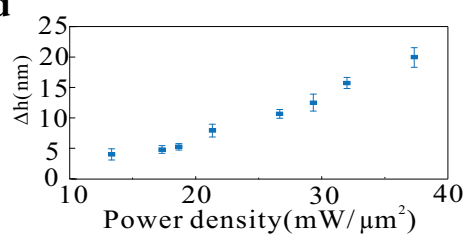

g h

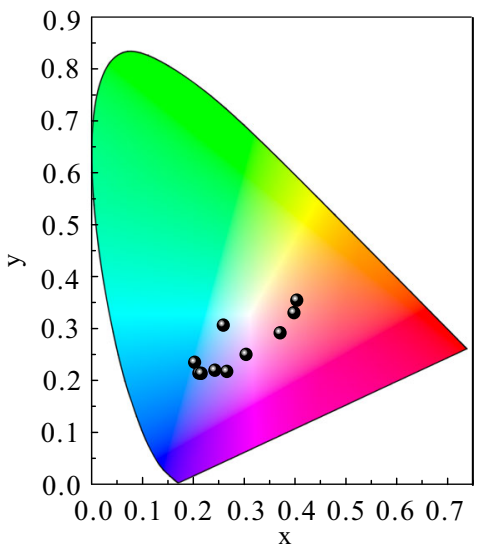

Fig. 2 Laser exfoliating $\mathbf{M o S}_{\mathbf{2}}$ thin films with nanometric thickness precisions. a The AFM image of laser thinned regions obtained at different laser doses. $\mathbf{b}$ The height change corresponding to the red dotted line in (a). The height of the steps is about $3 \mathrm{~nm}$. $\mathbf{c}$ The variation of characteristic Raman spectra of $\mathrm{MoS}_{2}$ flakes as the increase of laser powers. $\mathbf{d}$ Extracted thickness change from Raman spectra at corresponding laser powers. e, $\mathbf{f}$ Reflection spectra of $\mathrm{MoS}_{2}$ thin films prepared on the Au substrate with different nanometric thicknesses. $\mathbf{g}, \mathbf{h}$ Experimentally obtained reflection color palettes and color coordinate diagram through laser exfoliating at variant powers.
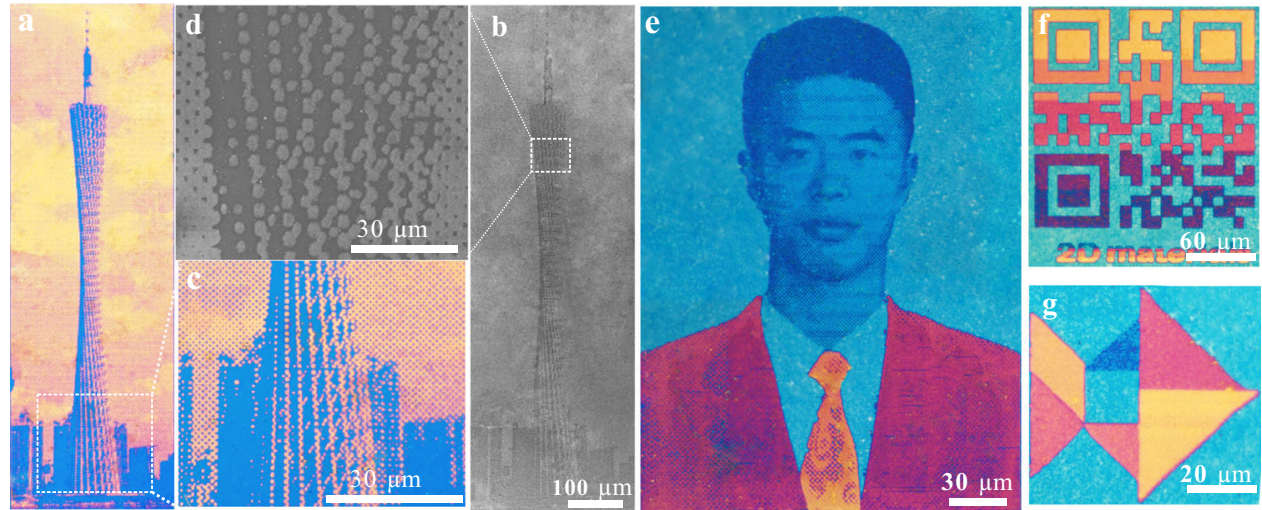

Fig. 3 High fidelity color images by laser exfoliated nanometric flat prints. a, c Are optical microscopic image and zoom-in view. b, d Are SEM image and sectional zoom-in view. A collection of color images printed by the laser exfoliation method with atomic thickness precisions and subwavelength feature sizes in continuous tone $(\mathbf{f}, \mathbf{g})$ and half tone modes $(\mathbf{a}, \mathbf{e})$ demonstrates the capability of high color fidelity and high spatial resolution.

Flat prints and binocular stereoscopic images. The ultrasensitive resonance control allows the generation of nanometric flat prints for color images $28-31$ (Supplementary Fig. 8). Figure $3 \mathrm{a}-\mathrm{e}$ are collections of halftone images patterned by laser writing methods. The color halftone effect is realized through controlling the printed pixel density. The underlying mechanism of laser exfoliation can be attributed to photothermal effects, where the local heating effect and heat diffusion can be flexibly controlled by the focusing conditions to pattern subwavelength feature sizes. By dexterous control the laser recipe, the patterned image composed of subwavelength scale pixels can achieve a high spatial resolution up to $58,000 \mathrm{dpi}$. The minimum pixel size is measured around $400 \mathrm{~nm}$ given a focusing lens with a numerical aperture of 0.75 . To demonstrate the up-scalability, a Canton Tower with a millimeter scale was printed with a high resolution and high fidelity (Fig. 3a). Scanning electron microscope (SEM) image of the prints is depicted in Fig. 3d. The SEM image and optical micrograph of the selected region with fine details are shown in Fig. $3 b, c$, respectively. Figure $3 \mathrm{f}-\mathrm{g}$ show another example of images or even color $\mathrm{QR}$ codes printed with 
continuous tone. More examples of color images generated by nanometric flat pigments can be seen in Supplementary Fig. 9. Because the ultra-sensitive resonance stems from interfacial phase shifts which are immune to the incident angle, the color images demonstrate an angle robust performance (Supplementary Fig. 10). Detailed performance was further compared with other structural color literature in Supplementary Table 2.

In addition to sensitive resonance wavelength modulation to generate flat color prints, this nanometric thickness dependent resonance control opens up efficient diffraction effects based on strong amplitude modulations to shape the impinging wavefronts. Supplementary Fig. 11 illustrates the measured diffraction efficiency of patterned microgratings with a period of $1000 \mathrm{~nm}$ and a thickness corrugation of $10 \mathrm{~nm}$ as a function of wavelengths. Given the ultra-thin nanometric thickness of 20 $\mathrm{nm}$ of the top $\mathrm{MoS}_{2}$ thin films, the first-order diffraction efficiency (defined as the first-order intensity divided by summed intensities of first-order and zeroth-order) can reach up to $36 \%$ at the optimal wavelength of $560 \mathrm{~nm}$, which is sufficient to develop a binocular stereoscopic view approach by multi-perspective diffractive images. The principle and the configuration are schemed in Fig. 4a, b. Two perspective images projected at two different directions were encoded into two sets of amplitudemodulated diffractive gratings with different periods of $1200 \mathrm{~nm}$ and $1800 \mathrm{~nm}$, respectively (Supplementary Fig. 12). By horizontally interleaving the two sets of laser printed gratings to form a $10 \mu \mathrm{m}$ sized "pixel", two perspective images can be diffracted and projected to different directions corresponding to left and right eyes at an oblique incidence (Fig. 4b). Figure 4c, d show the captured portrait images taken from different perspective that can form a binocular stereoscopic view. The zoom-in views of the SEM image and optical micrograph of the laser printed interleaved grating structures are shown in Fig. 4e, f.

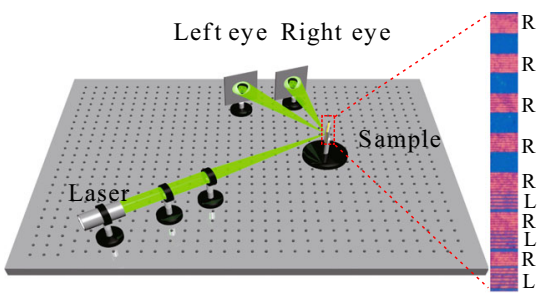

b
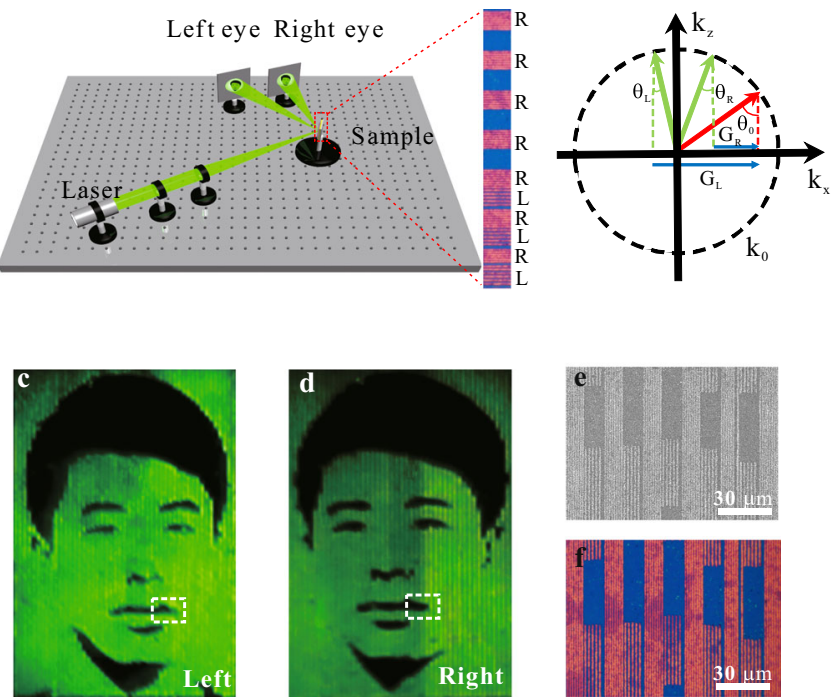

Fig. 4 Laser exfoliated nanometric flat diffractive prints for binocular stereoscopic images. a The optical configuration for binocular stereoscopic images. The inset shows optical microscopic images of laser exfoliated nanometric flat diffractive pixels made of two sets of grating structures. $\mathbf{b}$ Design principle of stereoscopic views by diffracting light from binocular positions $\left(\theta_{L}\right.$ and $\left.\theta_{R}\right)$ at oblique incident angles $\left(\theta_{0}\right)$. The dashed circles with radius $k_{0}=2 \pi / \lambda$ represent the wave vector of light. $\mathbf{c}, \mathbf{d}$ are different images acquired by the left and right eyes, which were false-color images of the human face taken from different angles, respectively. e, f Enlarged views of optical microscopic image and corresponding SEM image in the white dotted boxes in (c, d).

\section{Discussions}

By removing complex and sophisticated electron beam nanolithography procedures that are heavily resorted to, the demonstrated laser writing methods with atomic thickness precisions and subwavelength feature sizes paves the way to on-demand fabrication of ultra-thin TMD based opto-electronic devices with great facility and up-scalability. By introducing the interfacial phase shifts, the exotic dispersion of TMD materials empowers an ultra-sensitive light field manipulation scheme and opens avenues to a class of nanometric flat optical elements. Furthermore, the proof-of-principle demonstration of flat color prints and further multi-perspective stereoscopic views base on such nanometric amplitude-modulated diffractive pixels provides a viable scheme to construct light field manipulation based flat optics with ultracompact footprints and superior miniaturization. Combing their layer-dependent behaviors of bandgaps, the ultra-sensitive light field management in resonance control by the nanometric thickness of TMD thin films can dramatically push the state-ofthe-art and nourish multifarious functionalities of emerging $2 \mathrm{D}$ integrated opto-electronic devices.

\section{Methods}

Sample preparation. The $\mathrm{MoS}_{2}$ thin film with a lateral size of several millimeters was firstly deposited onto $\left.\mathrm{Si}_{2} / \mathrm{SiO}_{2}(300 \mathrm{~nm} \mathrm{SiO})_{2}\right)$ substrate with pulse laser deposition (PLD) method, and then was transferred onto a gold substrate obtained by heat evaporation. In the PLD process, a KrF laser with $248 \mathrm{~nm}$ wavelength was used and the commercial $\mathrm{MoS}_{2}$ target. The condition of fabrication $\mathrm{MoS}_{2}$ layer was $10^{-5}$ pressure and 780 degree. After the deposition, the pressure was maintained and annealed for $5 \mathrm{~min}$ at the same condition, and then the temperature was decreased at a rate of $10^{\circ} \mathrm{C} / \mathrm{min}$ to the room temperature. The power of the laser pulse was $150 \mathrm{~mJ}$ with a repeat frequency of $5 \mathrm{~Hz}$. The thickness of the $\mathrm{MoS}_{2}$ layer was controlled by the pulse number of the laser. The $\mathrm{MoS}_{2}$ film was transferred onto the gold substrate with wet transfer method, where a polymethylmethacrylate (PMMA) layer was spinned onto the $\mathrm{Si} / \mathrm{SiO}_{2} / \mathrm{MoS}_{2}$ films with a spin rate of $500 \mathrm{rmp}$ for $10 \mathrm{~s}$ and then $3000 \mathrm{rpm}$ for $60 \mathrm{~s}$. And then, the PMMA layer was immobilized through baking for $10 \mathrm{~min}$ at a temperature of $100^{\circ} \mathrm{C}$. After this, the sample was immersed in $\mathrm{KOH}$ solution to remove the substrate and the $\mathrm{MoS}_{2}$ with PMMA film float onto the solution surface. Then the residual $\mathrm{KOH}$ was washed by putting the $\mathrm{MoS}_{2}$ and PMMA film in deionized water for several seconds. The $\mathrm{MoS}_{2}$ and PMMA film was then spread onto the gold substrate and baked for $10 \mathrm{~min}$ under a temperature of $95^{\circ} \mathrm{C}$. Then the PMMA layer was removed by immersing in acetone, ethanol and deionized water for $30 \mathrm{~s}$, respectively.

Laser printing. The samples were placed on a computer-controlled $3 \mathrm{D}$ translation stage. The continuous wave laser beam at the wavelength of $532 \mathrm{~nm}$ was attenuated and focused by an objective lens $(\times 50,0.75 \mathrm{NA})$. The effective exposure time is $4.33 \mathrm{~ms}$. The beam power was adjusted to the desired value with a neutral density attenuator. During the laser printing, the movement of the translation stage was synchronized with the laser shutter to control the exposure of irradiances. The authors affirm that human research participants provided informed consent for publication of the images in Figs. 3, 4, and S9.

Sample characterization and optical measurements. Reflection color images of the sample were characterized using an objective lens (MPlanFL $\mathrm{N}, \times 50 \times / 0.8$, Olympus Co.). A CCD camera (Olympus, BX53, Olympus Co) was used to acquire the images from the sample. The spectra were characterized with a home built confocal microscope coupled to a spectrometer (Andori500). The sample was illuminated using a halogen white light source using an objective lens (MPlanFL N, $\times 20 \times / 0.45$, Olympus Co.). The reflected light was collected through the same objective lens and recorded using a spectrometer. The reflected intensity was normalized by the spectrum of the lamp obtained by reflection measurements with a silver mirror.

Characterization. An atomic force microscope (Ntegra solaris, NT-MDT Spectrum Instruments, Moscow, Russia) has been used to study the topography and determine the height of patterned $\mathrm{MoS}_{2}$ flakes. A Raman spectrometer (RENISHAW inVia) was used in a backscattering configuration excited with a visible laser beam $(\lambda=532 \mathrm{~nm}$, power $5 \mathrm{mw})$ to confirm the layer number of $\mathrm{MoS}_{2}$ flakes.

The layered structure is verified by X-ray diffraction (XRD) and high-resolution transmission electron microscopy (TEM) experiments. The sample is a crosssection sample cut with FIB (FEI Quanta 3D FEG). TEM images and Local energydispersive X-ray spectroscopy (EDX) was carried on a FEI Tecnai F20 S-TWIN operated at $200 \mathrm{kV}$. High-resolution ADF-STEM were performed in a probecorrected STEM (FEI Titan Chemi STEM) operated at $200 \mathrm{kV}$. For illumination 
and in situ fabricatior, a convergence angle of $21.4 \mathrm{mrad}$, a probe current of $\sim 70 \mathrm{pA}$, a range of acceptance angle of ADF detector was 43.4-200 mrad, and a pixel dwell time of $10 \mu \mathrm{s}$.

Numerical calculations. The reflection and transmission coefficients from a single interface is calculated by using the Fresnel equations, $r_{i j}=\left(p_{i}-p_{j}\right) /\left(p_{i}+p_{j}\right) r_{i j}=$ $\left(p_{i}-p_{j}\right) /\left(p_{i}+p_{j}\right), t_{i j}=2 p_{i} /\left(p_{i}+p_{j}\right) t_{i j}=2 p_{i} /\left(p_{i}+p_{j}\right), p_{i}=n_{i} \cos \left(\theta_{i}\right)$. Here, $t_{i j}$ and $r_{i j}$ are transmission and reflection coefficients under illumination from medium $i$ to medium $j, n_{i}$ is the complex refractive index of medium $i$ and $\theta_{i}$ is the angle between the propagating direction of the light wave within the medium and the normal direction of the layered films. The reflection phase shift from the interfaces was extracted from the reflection coefficients and then were added up to the total phase shift. The reflection from the air-FP-substrate layers was directly calculated by using $r=r_{12}+\frac{t_{12} t_{21} r_{23} e^{-i 2 q}}{1-r_{21} r_{23} e^{-12 \varphi}}$, where 1,2,3 represent the air, FP layer, and substrate, respectively, $\varphi$ is the propagation phase accumulation in the FP layer.

\section{Data availability}

The data that support the plots within this paper and other findings of this study are available from the corresponding author upon reasonable request.

Received: 26 January 2020; Accepted: 29 January 2021;

Published online: 19 February 2021

\section{References}

1. Geim, A. K. \& Novoselov, K. S. The rise of graphene. Nat. Mater. 6, 183-191 (2007).

2. Novoselov, K. S. et al. Electric field effect in atomically thin carbon films. Science 306, 666-669 (2004).

3. Wang, Q. H., Kalantar-Zadeh, K., Kis, A., Coleman, J. N. \& Strano, M. S Electronics and optoelectronics of two-dimensional transition metal dichalcogenides. Nat. Nanotechnol. 7, 699 (2012).

4. Mak, K. F., Lee, C., Hone, J., Shan, J. \& Heinz, T. F. Atomically thin MoS 2: a new direct-gap semiconductor. Phys. Rev. Lett. 105, 136805 (2010).

5. Lopez-Sanchez, O., Lembke, D., Kayci, M., Radenovic, A. \& Kis, A. Ultrasensitive photodetectors based on monolayer MoS 2. Nat. Nanotechnol. 8, 497 (2013).

6. Lee, H. S. et al. MoS2 nanosheet phototransistors with thickness-modulated optical energy gap. Nano Lett. 12, 3695-3700 (2012).

7. Mak, K. F., He, K., Shan, J. \& Heinz, T. F. Control of valley polarization in monolayer $\mathrm{MoS}_{2}$ by optical helicity. Nat. Nanotechnol. 7, 494 (2012).

8. Radisavljevic, B., Radenovic, A., Brivio, J., Giacometti, V. \& Kis, A. Single-layer $\mathrm{MoS}_{2}$ transistors. Nat. Nanotechnol. 6, 147-150 (2011).

9. Lembke, D. \& Kis, A. Breakdown of high-performance monolayer $\mathrm{MoS}_{2}$ transistors. ACS nano 6, 10070-10075 (2012).

10. Bertolazzi, S., Krasnozhon, D. \& Kis, A. Nonvolatile memory cells based on $\mathrm{MoS}_{2}$ /graphene heterostructures. ACS nano 7, 3246-3252 (2013).

11. Radisavljevic, B., Whitwick, M. B. \& Kis, A. Integrated circuits and logic operations based on single-layer MoS2. ACS nano 5, 9934-9938 (2011).

12. Yang, J. et al. Atomically thin optical lenses and gratings. Light.: Sci. Appl. 5, e16046 (2016).

13. Liu, H.-L. et al. Optical properties of monolayer transition metal dichalcogenides probed by spectroscopic ellipsometry. Appl. Phys. Lett. 105, 201905 (2014).

14. Verre R. et al. Transition metal dichalcogenide nanodisks as high-index dielectric Mie nanoresonators. Nature Nanotechnol. 14, 679-683 (2019).

15. Liu, C.-H. et al. Ultrathin van der Waals metalenses. Nano Lett. 18, 6961-6966 (2018).

16. Coleman, J. N. et al. Two-dimensional nanosheets produced by liquid exfoliation of layered materials. Science 331, 568-571 (2011).

17. Smith, R. J. et al. Large-scale exfoliation of inorganic layered compounds in aqueous surfactant solutions. Adv. Mater. 23, 3944-3948 (2011).

18. Han, G. H. et al. Laser thinning for monolayer graphene formation: heat sink and interference effect. ACS nano 5, 263-268 (2010).

19. $\mathrm{Li}, \mathrm{X}$. et al. Athermally photoreduced graphene oxides for three-dimensional holographic images. Nat. Commun. 6, 6984 (2015).

20. Li, X., Lan, T.-H., Tien, C.-H. \& Gu, M. Three-dimensional orientationunlimited polarization encryption by a single optically configured vectorial beam. Nat. Commun. 3, 998 (2012).

21. Rui, Z. et al. Enhancement of laser ablation via interacting spatial double-pulse effect. OEA 1, 180014 (2018).
22. Yeh P. Optical Waves in Layered Media (Wiley Online Library, 1988).

23. Kats, M. A., Blanchard, R., Genevet, P. \& Capasso, F. Nanometre optical coatings based on strong interference effects in highly absorbing media. Nat. Mater. 12, 20-24 (2013).

24. Kandemir, A., Yapicioglu, H., Kinaci, A., Çağın, T. \& Sevik, C. Thermal transport properties of $\mathrm{MoS}_{2}$ and $\mathrm{MoSe}^{2}$ monolayers. Nanotechnology 27, 055703 (2016).

25. Kang, J. et al. Thickness sorting of two-dimensional transition metal dichalcogenides via copolymer-assisted density gradient ultracentrifugation. Nat. Commun. 5, 5478 (2014).

26. Lee, C. et al. Anomalous lattice vibrations of single-and few-layer $\mathrm{MoS}_{2}$. ACS nano 4, 2695-2700 (2010).

27. Castellanos-Gomez, A. et al. Laser-thinning of $\mathrm{MoS}_{2}$ : on demand generation of a single-layer semiconductor. Nano Lett. 12, 3187-3192 (2012).

28. Kumar, K. et al. Printing colour at the optical diffraction limit. Nat. Nanotechnol. 7, 557 (2012).

29. Tan, S. J. et al. Plasmonic color palettes for photorealistic printing with aluminum nanostructures. Nano Lett. 14, 4023-4029 (2014).

30. Zhang, Y. et al. Full-visible multifunctional aluminium metasurfaces by in situ anisotropic thermoplasmonic laser printing. Nanoscale Horiz. 4, 601-609 (2019).

31. $\mathrm{Hu}, \mathrm{D}$. et al. Laser-splashed three-dimensional plasmonic nanovolcanoes for steganography in angular anisotropy. ACS Nano 12, 9233-9239 (2018).

\section{Acknowledgements}

The authors are grateful for the support of STEM sample fabrication and characterization by Ms. Li Gu and Prof. Chuanhong Jin in Zhejiang University. This research was supported by National Key R\&D Program of China (2018YFB1107200), National Natural Science Foundation of China (NSFC) (Grant 61705084), Guangdong Provincial Innovation and Entrepreneurship Project (Grant 2016ZT06D081).

\section{Author contributions}

D.H. and H.L. contributed equally to this work. X.L. and B.L. conceived the idea and supervised the project. D.H., H.L., and Y.L. performed the experiments. H.L. and H.J. assisted spectral measurements. Y.Z. and S.X. prepared the sample. D.H. and H.L. performed the modelling and simulation. D.H., H.L., J.Z., B.G., B.L. Y.C., and X.L. analyzed data and prepared the manuscript. All authors contributed to the discussion and manuscript writing.

\section{Competing interests}

The authors declare no competing interests.

\section{Additional information}

Supplementary information The online version contains supplementary material available at https://doi.org/10.1038/s41467-021-21499-4.

Correspondence and requests for materials should be addressed to Y.C., L.B. or X.L.

Peer review information Nature Communications thanks Debashis Chanda, Teun-Teun Kim, and the other, anonymous, reviewer(s) for their contribution to the peer review of this work. Peer review reports are available.

Reprints and permission information is available at http://www.nature.com/reprints

Publisher's note Springer Nature remains neutral with regard to jurisdictional claims in published maps and institutional affiliations.

\begin{abstract}
Open Access This article is licensed under a Creative Commons Attribution 4.0 International License, which permits use, sharing, adaptation, distribution and reproduction in any medium or format, as long as you give appropriate credit to the original author(s) and the source, provide a link to the Creative Commons license, and indicate if changes were made. The images or other third party material in this article are included in the article's Creative Commons license, unless indicated otherwise in a credit line to the material. If material is not included in the article's Creative Commons license and your intended use is not permitted by statutory regulation or exceeds the permitted use, you will need to obtain permission directly from the copyright holder. To view a copy of this license, visit http://creativecommons.org/ licenses/by/4.0/.
\end{abstract}

() The Author(s) 2021 